\title{
əMapping Altimetry in the Forthcoming SWOT Era by Back-and-Forth Nudging a One-Layer Quasigeostrophic Model $\mathscr{O}$
}

\author{
Florian Le Guillou, ${ }^{\mathrm{a}}$ Sammy Metref, ${ }^{\mathrm{a}}$ Emmanuel Cosme, ${ }^{\mathrm{a}}$ Clément Ubelmann, ${ }^{\mathrm{b}}$ Maxime Ballarotta, ${ }^{\mathrm{c}}$ \\ JULIEN LE SOMMER, ${ }^{\mathrm{a}}$ AND JACQUES VERRON ${ }^{\mathrm{b}}$ \\ ${ }^{a}$ Université Grenoble Alpes, CNRS, IRD, Grenoble INP, IGE, Grenoble, France \\ ${ }^{\mathrm{b}}$ Ocean Next, Grenoble, France \\ ${ }^{\mathrm{c}}$ Collecte Localisation Satellites, Ramonville-Saint-Agne, France
}

(Manuscript received 29 June 2020, in final form 29 September 2020)

\begin{abstract}
During the past 25 years, altimetric observations of the ocean surface from space have been mapped to provide two dimensional sea surface height (SSH) fields that are crucial for scientific research and operational applications. The SSH fields can be reconstructed from conventional altimetric data using temporal and spatial interpolation. For instance, the standard Developing Use of Altimetry for Climate Studies (DUACS) products are created with an optimal interpolation method that is effective for both low temporal and low spatial resolution. However, the upcoming nextgeneration SWOT mission will provide very high spatial resolution but with low temporal resolution. The present paper makes the case that this temporal-spatial discrepancy induces the need for new advanced mapping techniques involving information on the ocean dynamics. An algorithm is introduced, dubbed the BFN-QG, that uses a simple data assimilation method, the back-and-forth nudging (BNF), to interpolate altimetric data while respecting quasigeostrophic (QG) dynamics. The BFN-QG is tested in an observing system simulation experiments and compared to the DUACS products. The experiments consider as reference the high-resolution numerical model simulation NATL60 from which are produced realistic data: four conventional altimetric nadirs and SWOT data. In a combined nadirs and SWOT scenario, the BFN-QG substantially improves the mapping by reducing the root-mean-square errors and increasing the spectral effective resolution by $40 \mathrm{~km}$. Also, the BFN-QG method can be adapted to combine large-scale corrections from nadir data and small-scale corrections from SWOT data so as to reduce the impact of SWOT correlated noises and still provide accurate SSH maps.
\end{abstract}

KEYWORDS: Sea/ocean surface; Altimetry; Data assimilation

\section{Introduction}

Gridded maps of sea surface height (SSH) are widely used to study mesoscale ocean dynamics and their impact on global climate (Morrow and Le Traon 2012). SSH maps enable the tracking of mesoscale eddies (Chelton et al. 2011) and the study of their variability in space and time (Pascual et al. 2006). This leads to better quantification of the transport of kinetic energy, heat, and salt, which are to a large extent driven by mesoscale eddies (Fu et al. 2010). SSH maps make it possible to quantify sea level rise at global and regional scales (Ablain et al. 2017), are also used for operational monitoring of the marine environment (von Schuckmann et al. 2018) and are fundamental for data-driven reconstructions of the $3 \mathrm{D} d y-$ namics as those provided by the Copernicus service (e.g., Mulet et al. 2012; Buongiorno Nardelli 2020).

๑ Denotes content that is immediately available upon publication as open access.

Supplemental information related to this paper is available at the Journals Online website: https://doi.org/10.1175/JTECH-D-200104.s1.

Corresponding author: Florian Le Guillou, florian.leguillou@ univ-grenoble-alpes.fr
The most commonly used SSH maps, the Developing Use of Altimetry for Climate Studies (DUACS) products, are derived from a statistical space-time interpolation of nadir altimeter observations. This intrinsically limits the effective resolution [as defined in Skamarock (2004), i.e., the fully resolved scales] of DUACS SSH maps to $150-200 \mathrm{~km}$ at middle latitudes (Ballarotta et al. 2019). The SSH mapping algorithm was developed by CNES and CLS in 1997, as part of the DUACS project, and has been continuously improved since then (Taburet et al. 2019). The DUACS products are now distributed by the Copernicus Marine Environment Monitoring Service (CMEMS). DUACS algorithm implements a statistical interpolation of SSH satellite data in space and time to produce global daily maps (Le Traon et al. 1998). The data are collected by a constellation of 2 to 4 nadir-looking altimeters (sometimes referred to as conventional altimeters), and characterized by large data gaps reaching $200 \mathrm{~km}$ in the zonal direction at the equator.

The Surface Water and Ocean Topography (SWOT; Fu et al. 2012; Morrow et al. 2019) altimetry mission, to be launched in early 2022, will open the way to SSH maps with resolution significantly higher than $150 \mathrm{~km}$ at midlatitudes, but this perspective entails a thorough revisit of the mapping algorithm. SWOT will considerably increase the measurement density at the surface of the oceans thanks to SSH measurements at a kilometric pixel resolution over a swath $120 \mathrm{~km}$ wide. On the swath, SWOT is expected to resolve scales down to $15 \mathrm{~km}$ at low latitude and $30-45 \mathrm{~km}$ at mid- and high 
latitudes (Wang et al. 2019). In its science phase, SWOT will have a 21 days repeat orbit, allowing an average revisit time of 11 days in most of the globe. Some of the dynamical processes observable by SWOT evolve over time scales on the order of 1 day, much shorter than the satellite revisit time. Consequently, the mapping method implemented in the current DUACS system will certainly not be sufficient to draw the maximum benefit from SWOT. A linear interpolation will filter most of the observed small-scale signals between two passes of the satellite, as anticipated by Gaultier et al. (2016).These authors advocate for using more advanced methods to build SSH maps.

One particularly appealing option to derive SSH maps from SWOT and nadir altimetry is to introduce information on ocean dynamics in the mapping algorithm or, worded differently, combine altimetry and a numerical model of the ocean dynamics using some data assimilation technique. The model provides a full, 2D gridded representation of SSH, though not always consistent with observations. In a data assimilation process, it acts as a physical interpolator or extrapolator of observations in space and time. Data assimilation aims at melding model data and observations to produce full, 2D gridded $\mathrm{SSH}$ maps consistent with observations and model dynamics at once. Assimilation methods exhibit a wide range of complexity and diversity of applications. The method often referred to as the simplest one is nudging (Anthes 1974). Nudging consists of introducing an extra term in the model prognostic equations, to elastically pull the simulated variables toward the observations. Several pioneering experiments of altimetry assimilation actually started with nudging (e.g., Holland and Malanotte-Rizzoli 1989; Verron 1990, 1992; Blayo et al.1994). Much more advanced assimilation methods exist to efficiently assimilate altimetric data in complex, general circulation models (e.g., Leeuwenburgh 2005; Martin et al. 2007; Lea et al. 2008; Wan et al. 2010; Storto et al. 2011). These methods are often designed to estimate the full, 3D and multivariate state of the ocean, and tend to be computationally expensive. A belief shared among the authors and driving the choices of tools for this work is that the best method is the one that best responds to the problem posed, and this is not always the most sophisticated one.

The relevance of using a simpler dynamical framework has been shown in Ubelmann et al. (2015). Although the model considered is representing a very reduced dynamic (based on the conservation of potential vorticity), the quasigeostrophic (QG) dynamical framework is able to capture a large part of the mesoscale dynamics for small Rossby numbers (Vallis 2006). As a proof of concept, Ubelmann et al. (2015) reconstructed the trajectory between two SSH fields 10 days apart from each other by averaging a model forward run (initialized by the first snapshot) and a backward run (initialized by the second snapshot). They show a significant improvement of the two dimensional reconstruction of SSH fields compared with a linear interpolation (such as the one implemented in the DUACS system). The method was then successfully applied with synthetic nadir altimeter data (Ubelmann et al. 2016) and finally in a more operational context with real data (Ballarotta et al. 2020).
Motivated by the conclusions drawn in this series of papers, we investigate an algorithm, dubbed the BFN-QG, based on a 1.5-layer QG model and a data assimilation method called the back-and-forth nudging (BFN; Auroux and Blum 2008) to create high-resolution SSH maps from nadir and SWOT altimetry. The goal of the studied algorithm is to apply a QG dynamical constraint to the data interpolation, using a conceptually simple and computationally reasonable data assimilation smoothing method. The standard nudging method adds to the model equations a feedback term which is a tunable nudging coefficient multiplied by the difference between the observation and the equivalent model quantity (Hoke and Anthes 1976). The backward nudging applies the same procedure on the backward-in-time model equations and the nudging term with opposite sign. The BFN iteratively combines the standard and the backward nudging to provide a smoothing over a time period of the best fit to the observations under the dynamical constraint (Auroux and Blum 2008). In the present application, the QG model can be seen as a weak constraint to the altimetric data interpolation.

The goal of this paper is to evaluate the SSH mapping abilities of the BFN-QG in comparison with the interpolation method, presently used in DUACS, with conventional altimetry and SWOT data. The methods are tested with simulated altimetric observations realistically distributed in space and time using an observing system simulation experiments (OSSE) approach. The reference simulation (Nature Run) is a very high-resolution ocean simulation of the North Atlantic (NATL60; Molines 2018) from which simulated SSH observations are built considering four nadir and one SWOTlike virtual altimeter sampling along realistic orbits. The focus is made on one region of the Gulf Stream characterized by strong mesoscale dynamics. Three observational scenarios are investigated: the four nadirs only (Nadirs), the SWOT data only (SWOT) and the combination of the four nadirs and the SWOT data (Nadirs + SWOT). For the SWOT data, in the latter two scenarios, an alternative implementation of the BFN-QG where the nudging is applied to the relative vorticity only-named $\operatorname{SWOT}(\xi)$ in opposition to the standard nudging on the potential vorticity $\operatorname{SWOT}(q)$ that includes both relative vorticity and vortex stretching - is also investigated in anticipation of possibly strong correlated SWOT errors. This alternative implementation is expected to reduce the impact of correlated SWOT errors on the SSH reconstruction. In each case, a systematic comparison with the DUACS products (made from the same synthetic dataset) is carried out to evaluate the relative performance of the new mapping technique in terms of accuracy and spectral resolution.

The paper is structured as follows. Section 2 details the data assimilation method, from the theoretical aspects to the strategical implementation. Section 3 presents the experimental design, based on OSSE, to assess the ability of the BFN-QG to derive SSH maps from SWOT and nadir altimetry. In section 4, we evaluate the performances of the BFN-QG technique in comparison with DUACS products. Conclusions and perspectives are drawn in section 5 . 


\section{Data assimilation technique}

\section{a. Dynamical model}

In Ubelmann et al. (2015), the authors have shown the relevance of PV conservation as a weak dynamical constrain to propagate high-spatial-resolution SSH data in time. Thus, we decide to use the same QG model as in Ubelmann et al. (2015) to dynamically propagate in space and time the altimetric data. The model considers 1.5 layers and no atmospheric forcing. The reconstructed circulation is then the combination of observations, QG dynamics, and boundary conditions only. Note that the collapse of energy due to numerical dissipation and the absence of atmospheric forcing is compensated by data assimilation.

The adiabatic QG dynamics in the 1.5-layer model are governed by the conservation of PV, $q$ (Vallis 2006):

$$
\frac{\partial q}{\partial t}+J(\psi, q)=0
$$

where $J$ is the Jacobian operator and the streamfunction $\psi$ is proportional to SSH:

$$
\psi=\frac{g}{f} \mathrm{SSH}
$$

with $g$ the gravity constant, and $f$ the Coriolis parameter. The $\mathrm{PV}$ is linked to the streamfunction by the elliptical equation:

$$
q=\nabla^{2} \psi-\frac{1}{L_{\mathrm{R}}^{2}} \psi,
$$

where $\nabla^{2}$ is the Laplace operator and $L_{R}$ is the first baroclinic Rossby radius of deformation.

A few properties of the QG model are worth being emphasized to anticipate the assimilation methodology. Two terms appear in the expression of PV. The first one $\left(\nabla^{2} \psi\right)$, called the relative vorticity $(\xi)$, is the vorticity of the fluid as viewed in the rotating frame of Earth. The second one $\left(-1 / L_{\mathrm{R}}^{2} \psi\right)$, called vortex stretching, describes the mechanism of stretching of the fluid columns. There is an interplay between these two terms in the conservation of PV. The length scale on which relative vorticity and vortex stretching make equal contributions to $\mathrm{PV}$ is the first baroclinic Rossby radius of deformation $L_{R}$. The value of $L_{R}$ varies geographically (Chelton et al. 1998). It depends on Earth rotation, stratification, and water depth. We will see in section $2 \mathrm{c}$ that, depending on the type of sensor, one part, the other part, or the totality of the PV can be observed and therefore assimilated.

The time stepping from time $t_{i}$ to $t_{i+1}$ is implemented as follows. First, the streamfunction $\psi_{i}$ is computed by Eq. (2) and PV $q_{i}$ is diagnosed from Eq. (3). Then Eq. (1) is integrated in time to get $q_{i+1}$. Finally, $\psi_{i+1}$ (and therefore $\mathrm{SSH}_{i+1}$ ) is obtained by inverting the elliptical Eq. (3) using a gradient conjugate technique.

\section{b. The BFN algorithm}

The nudging technique is a conceptually simple data assimilation method first employed in the meteorological context (Hoke and Anthes 1976). It consists in adding a feedback term to the prognostic equation of the model. The feedback term is proportional to the difference between the observations and the state of the model. In our study, the prognostic equation is Eq. (1) and the state variable of the model is PV, $q$. To make the method description clear, we rewrite this equation as follows:

$$
\frac{\partial X}{\partial t}=\mathscr{L}(X, t)
$$

where $X$ is the state variable (here, PV) and $\mathscr{C}$ represents the physics of the model (here, the advection of PV by the surface flow).

Now, let us assume that observations $Y^{\text {obs }}$ of the model state are available in the time window $[0, T]$. These observations can be used to nudge the model, i.e., pull the state toward the observed trajectory.

\section{1) FORWARD NUDGING}

Assuming the initial state of the model $X_{0}$ is given, the forward nudging consists in resolving the system

$$
\left\{\begin{array}{l}
\frac{\partial X}{\partial t}=\mathscr{C l}(X, t)+K\left(Y^{\mathrm{obs}}-X\right), \quad 0 \leq t \leq T \\
X(0)=X_{0}
\end{array},\right.
$$

where $K$, called the nudging coefficient, has the dimension of the inverse of time. The value of $K$ determines the strength of the relaxation: the higher the value, the more the observation will impact the trajectory of the model.

\section{2) BACKWARD NUDGING}

Now, we assume that the initial condition is replaced by a final condition $X_{T}$. In our QG model, no irreversible phenomena, such as mixing, have been considered. It is therefore possible to propagate the model backward in time. In contrast to the forward nudging, the feedback term is added with an opposite sign (Auroux and Blum 2008):

$$
\left\{\begin{array}{l}
\frac{\partial X}{\partial t}=\mathscr{C l}(X, t)-K\left(Y^{\mathrm{obs}}-X\right), \quad T \geq t \geq 0 \\
X(T)=X_{T}
\end{array} .\right.
$$

\section{3) BFN ALGORITHM}

The BFN is the iterative combination of the forward nudging and the backward nudging over the time interval $[0, T]$. Assuming the initial state of the model is given, the forward nudging is first computed. The final state obtained by the forward propagation is then used as initial condition to run the backward nudging. The algorithm converges in several iterations toward a trajectory that both fits the observations and complies with the model dynamics (e.g., a maximum of 10 iterations were used in the following numerical experiments). This data assimilation method shares analogies with four-dimensional variational (4DVar) assimilation, in that both solve the problem of finding a model trajectory over a fixed-time interval, which best fits observations within this interval. The implementation of the BFN 

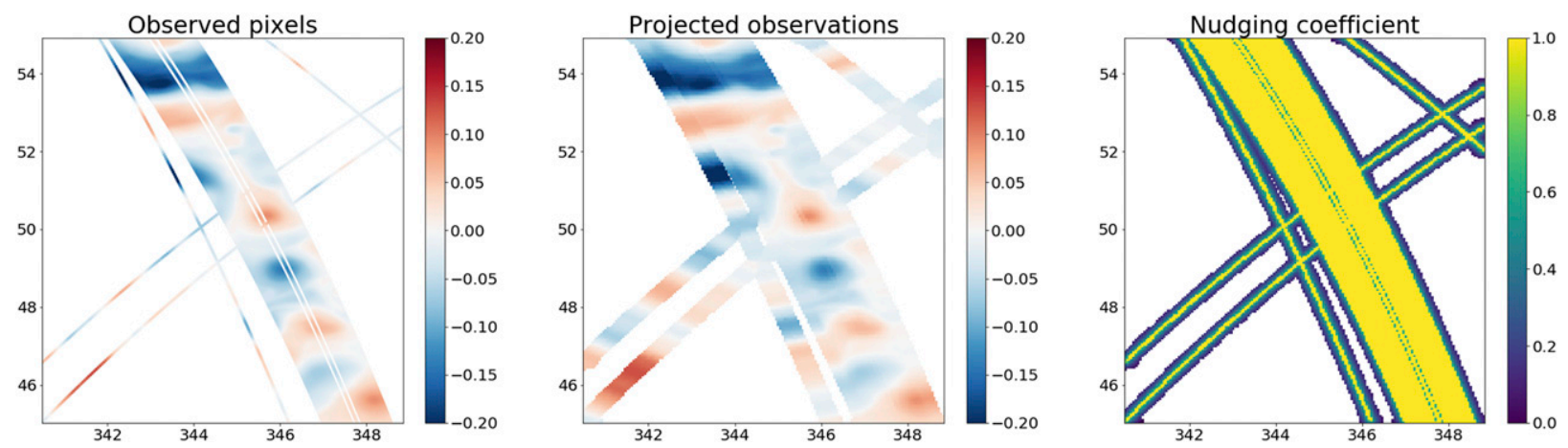

FIG. 1. (left) Available SSH (in m) measured by four nadir altimeters and SWOT in a $10^{\circ} \times 10^{\circ}$ region over a specific assimilation time window; (center) spatial influence of these observations; (right) related nudging term. White regions indicate (left) the absence of observations and (center),(right) the absence of nudging term.

is easier because it does not require the minimization tools needed by the 4DVar.

\section{c. Nudging strategy}

The nudging technique is relatively easy to implement if the assimilated data are direct observations of the prognostic variables, or if they can be conveniently projected onto the state space. Indeed, in Eqs. (5) and (6), the terms $Y^{\text {obs }}$ and $X$ (which refer, respectively, to the observations and to the prognostic variable) have to be of the same nature to be comparable. Depending on the state variable and the type of observations available, it is sometime necessary to use physical considerations to transform the observation or the state variable and compute the nudging term. For instance, in the adiabatic QG framework, the prognostic variable is PV, which is not directly observable. As mentioned previously, PV is the sum of two terms: the relative vorticity $\left(\nabla^{2} \psi\right.$ term) and the vortex stretching $\left(-1 / L_{\mathrm{R}}^{2} \psi\right.$ term).

In this study, the data to be nudged in the QG model are provided by conventional along-track (nadir) and SWOT SSH data.

Three possible options are then to nudge the full PV, which requires the computation of relative vorticity $\nabla^{2} \mathrm{SSH}$ from the observations, or to restrict the nudging to the vortex stretching component or to the relative vorticity component.

In this work, the nudging from nadir altimetry data is restricted to the vortex stretching component. These data being distributed along one-dimensional tracks, the two dimensional Laplace operator cannot be applied as is. Verron (1990) overtook this issue by assuming the relative vorticity to be isotropic. In this case, the Laplace operator reduces to $2 \partial^{2} / \partial^{2} s$ where $s$ is the along-track coordinate. Verron (1992) showed that nudging the relative vorticity is comparable to nudging SSH toward nadir altimetry measurements. To keep the formulation as simple as possible and save algorithm complexity, we implement the nudging toward SSH only.

With SWOT data, nudging is carried out with both relative vorticity and vortex stretching. Nudging the model full PV is possible, or the two PV components separately. An experiment motivated in section $3 c$ actually implements the nudging of relative vorticity only. The general form of the nudging term to be added to the prognostic Eq. (1) can be written as

$$
\begin{aligned}
K\left(Y^{\mathrm{obs}}-X\right)= & -\frac{1}{L_{R}^{2}} K_{\mathrm{SSH}}\left(\frac{g}{f} \mathrm{SSH}^{\mathrm{obs}}-\psi\right) \\
& +K_{\xi}\left[\nabla^{2}\left(\frac{g}{f} \mathrm{SSH}^{\mathrm{obs}}\right)-\nabla^{2}(\psi)\right],
\end{aligned}
$$

where $\mathrm{SSH}^{\text {obs }}$ is the observed SSH, $\psi$ the streamfunction of the model, $L_{R}$ the Rossby radius, $g$ the gravitational constant and $f$ the Coriolis parameter; $K_{\mathrm{SSH}}$ and $K_{\xi}$ are the nudging coefficients, to be adjusted. This formula holds for nadir observations, with $K_{\xi}=0$.

The abrupt nudging toward observations that are discrete in time and discontinuous in space can either generate strong gradients, leading to numerical instabilities, or apply a tooweak constraint on the model, leading to the absence of observational constraints. A common recipe to tackle this issue is to extend the space-time domain of influence of each observation (Ruggiero et al. 2015).

To compute the nudging terms (one for SWOT, one for nadir data), the observations are grouped together in assimilation time windows (one hour in this study). For each window presenting observations, the observation used to compute the nudging term (relative to SWOT or nadir data) at a model grid point is made from a weighted average of all observations occurring in the assimilation time window and within a spatial influence radius $D$ (to be prescribed). The weights are computed with the Gaspari-Cohn function (Gaspari and Cohn 1999), which mimics a Gaussian function with compact support. This means that the observations are spatially extended across the satellite tracks by a distance $2 D$. The nudging coefficient $K$ decreases exponentially as the distance from the track increases. Figure 1 illustrates a snapshot of available observations, their spatial influence, and the associated nudging coefficients.

Each nudging term, specific to one kind of sensor and one assimilation time window, is applied along a time window that is longer than the assimilation time window and centered on it. The length of this window is $2 \tau$ ( $\tau$ has to be prescribed). The nudging coefficient $K$ decreases exponentially as the distance 
(in time) from the middle of the assimilation time window increases.

Mathematically, for each nudging operation, the nudging coefficient at time $t$ and at the model grid point $x$ can be written as

$$
K(x, t)=K_{0} e^{-\left[\left(t-t_{a}\right) / \tau\right]^{2}} e^{-[d(x) / D]^{2}},
$$

where $K_{0}$ is the nominal value of the nudging coefficient and $t_{a}$ is the middle of the assimilation time window. $d(x)$ is the distance between the considered grid point and the observations. If this distance is below the grid resolution, it is set to 0 . In all the following experiments, $D$ is set to $10 \mathrm{~km}$. Parameters $K_{0}$ and $\tau$ change according to the type of observations and the variable nudged, as detailed in section $3 \mathrm{~b}$.

The BFN-QG time window is set to 7 days to reconstruct the dynamics over the three days in the middle. The window is then shifted by 3 days, allowing to estimate the dynamics over the next three days, and so on. The time window is prescribed from considerations on the observation sampling, the decorrelation time of the dynamical model, and computational complexity. On the one hand, this window should be long enough to include a sufficient number of observations and correctly constrain the dynamics, and make sure that the state estimations in the middle of the window are influenced by both past and future observations. On the other hand, the window must be reasonably short to limit computational complexity, and because a too-long window would make some observations useless at the window extremes. We estimate that 7 days are a good trade-off.

\section{Experimental design}

\section{a. Data}

In this study, we test the ability of the BFN-QG to dynamically interpolate altimetric observations by setting up twin experiments based on virtual observation data drawn from an ocean circulation model. First, we use a free model run that we consider as our ground truth [called Nature Run (NR)]. Second, the NR is used to generate synthetic altimetric observations from current (nadirs) and future (SWOT) observing systems. Then the synthetic observations are assimilated into the QG model by the BFN technique to produce gridded SSH maps that can be compared with the NR.

The NR has been drawn from the NATL60 configuration (Molines 2018) of the Nucleus for European Modelling of the Ocean (NEMO) model. With its $1 / 60^{\circ}$ horizontal resolution and 300 vertical levels over the North Atlantic basin, it is one of the most advanced basin-scale high-resolution simulation available today. For a detailed study dealing with physical characteristics of NATL60, we refer the reader to the paper of Ajayi et al. (2020). In short, these authors have shown that NATL60 exhibits a strong space-time variability in the size of small eddies $(<100 \mathrm{~km})$. The eddies are 2 times bigger in summer than in winter and their sizes vary geographical consistently with the latitudinal variation of the first Rossby radius of deformation. NATL60 has been used in several studies such as Metref et al. (2019, 2020), Fresnay et al. (2018), Amores

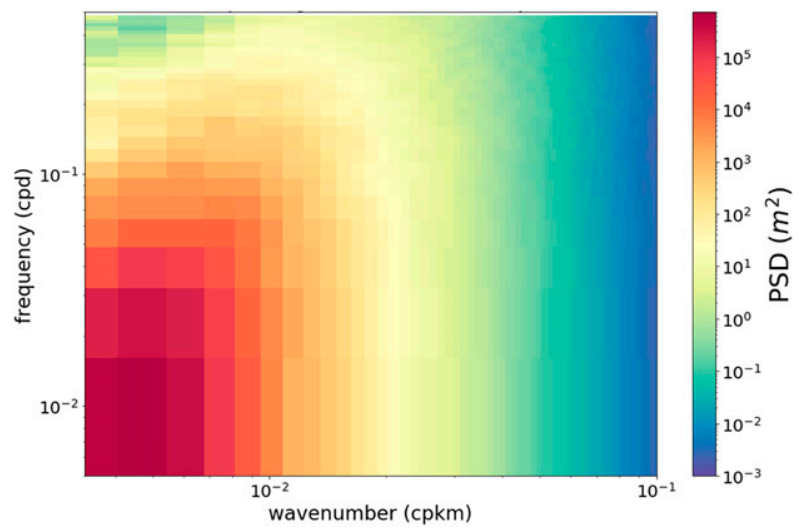

FIG. 2. SSH frequency-wavenumber spectra $\left(\right.$ in $\mathrm{m}^{2}$ ) of the studied region from 1 May to 1 Jul 2013.

et al. (2018) and Ajayi et al. (2019). NATL60 resolves finescale dynamical processes down to $15 \mathrm{~km}$ at the surface (Ajayi et al. 2020), allowing us to test the mapping capabilities in all scale ranges offered by SWOT.

The SSH field of this NR is subsampled by realistic synthetic satellite constellations, simulating both nadir (conventional) and SWOT altimetry. Existing simulator tools (Gaultier et al. 2016) are used to generate the datasets.

As a first baseline to represent current observing capabilities, we consider a constellation of four conventional nadir altimeters following the theoretical ground tracks of two interleaved Jason-like and Sentinel-3-like orbits. The data are extracted with the SWOT simulator tool (Gaultier et al. 2016) in its nadir mode, at an along-track resolution set to $6 \mathrm{~km}$ for each satellite. The data are considered perfect; i.e., no source of measurement error is simulated.

As a second main dataset of this study, we consider SWOTlike observations also generated with the SWOT simulator in its swath mode. The spatial resolutions are set to $2 \mathrm{~km}$, both in the along-track and across-track directions, consistently with the anticipated resolution of the future SWOT products. The observations provided by the Nadir module (which will be part of the payload) are also simulated, at a resolution of $6 \mathrm{~km}$. Here again, we do not consider any kind of measurement error. Yet it is clear that the measurement errors will considerably affect the quality of the signal. This is discussed in section 3c.

To provide a baseline for evaluating our new mapping technique, the DUACS method has been applied to the synthetic altimetry observations described previously. The parameters used for the computation are those presented in Taburet et al. (2019). The generated maps are regular in space $\left(0.25^{\circ} \times 0.25^{\circ}\right)$ and time (daily outputs).

\section{b. Configurations}

We focus on a region of the North Atlantic basin, containing a part of the Gulf Stream, dominated by energetic mesoscale dynamics. Figure 2 provides a description of the energy distribution both in space and time by showing the frequencywavenumber power spectral density. This figure reveals that the energy is continuously spread out over all frequencies and 
wavenumber, with the highest intensity for large space $(>100 \mathrm{~km})$ and time ( $>10$ days) scales. As the region is a $10^{\circ} \times 10^{\circ}$ box, the Rossby radius of deformation is assumed constant in the domain, which facilitates the implementation of the QG model. The prescribed value for the Rossby radius of deformation is based on Chelton et al. (1998): $30 \mathrm{~km}$.

To compare the performance of BFN-QG with the current mapping technique, the DUACS products, that were computed with the same artificial observations on the entire basin, are then extracted in the studied region over the same time period. This time period extends over three months, from 1 April to 1 July 2013. The first month is considered as a spinup period and is not considered in the diagnostics. Note that other experiments have been carried out in other time periods. The results, presented in the online supplemental material, show a strong robustness of the BFN-QG according to the dynamical regime.

Boundary conditions for the QG model are prescribed from the DUACS products. A relaxation zone, $1^{\circ}$ wide, induces a smooth integration of the boundary conditions into the inner region. The relaxation is performed with a Gaspari-Cohn function (Gaspari and Cohn 1999) taking the DUACS product value at the outer boundary, and the QG model value at the inner boundary of the relaxation zone.

The nudging parameters $\left(K_{0}\right.$ and $\tau$ ) are fixed after a set of sensitivity experiments (not shown here). The nominal nudging coefficient $K_{0}$ defines how strong the nudging is and the nudging temporal window $\tau$ defines how long the nudging is performed toward each observation. The retained values, listed in Table 1, are specific to each nudging operation (nudging on SSH or on $\xi$ ) and each kind of observations (nadirs or SWOT). Note that in Table 1 we fix the quantity $K_{0} d t$ ( $d t$ being the model time step) which has to be below 1 to ensure the numerical stability of Eqs. (5) and (6). As mentioned in section $2 c$, we nudge only the vortex stretching component of the PV toward nadir data.

Two aspects are worth mentioning to justify the choice of the retained values. First, as the relative vorticity reveals smaller structures than $\mathrm{SSH}, \tau$ and $K_{0}$ are set smaller for the nudging toward SWOT $(\xi)$ than toward SWOT (SSH). Second, $\tau$ is set higher for SWOT (SSH) than for Nadirs in order to compensate the poor temporal sampling of SWOT in this region.

Some experiments are also conducted nudging only relative vorticity toward SWOT data, as explained in section 3c.

\section{c. Observational scenarios}

The mapping techniques (DUACS and BFN-QG) are run in the studied region, over three months, and testing three different observation scenarios: (i) four nadir altimeters (Nadirs), (ii) SWOT alone (SWOT) and (iii) nadir altimeters and SWOT concomitantly (Nadirs + SWOT). It is recalled that DUACS and BFN-QG algorithms are run with the same synthetic observations, presented in section $3 \mathrm{a}$, to make the reconstructed fields comparable with each other.

To anticipate the processing of the real SWOT data, possibly affected by strong spatially correlated errors, two other BFN-QG experiments are introduced where the SWOT data are assimilated by the relative vorticity component of PV.
TABLE 1. Nudging parameters used in the experiments according to the kind of altimetric sensor (Nadir or SWOT) and the variable nudged (SSH or $\xi$ ).

\begin{tabular}{clc}
\hline \multicolumn{1}{c}{ Satellites } & $K_{0} d t$ & $\tau$ (days) \\
\hline Nadirs $(\mathrm{SSH})$ & 0.9 & 1 \\
SWOT $(\mathrm{SSH})$ & 0.9 & 2 \\
SWOT $(\xi)$ & 0.05 & 1 \\
\hline
\end{tabular}

The SWOT correlated errors, to a large part specific to the satellite and instrument design (Peral and Esteban-Fernandez 2018), are particularly challenging for the mapping of altimetry because they can present significant amplitudes but cannot be easily filtered out using conventional filters. Hopefully, these strong spatially correlated errors are expected to have a much lower signature on $\xi$ than on SSH (Gómez-Navarro et al. 2018). Ruling out the nudging of the vortex stretching component of $\mathrm{PV}$, and keeping only the relative vorticity component, is thus an option to tackle the correlated-error issue. Practically, this means that the SWOT observations is only used to nudge the second term of the right-hand side of Eq. (7). The two additional experiments nudging only the relative vorticity with SWOT are one experiment nudging SWOT only, SWOT $(\xi)$, and one experiment nudging SWOT and nadir altimeters, Nadirs $+\operatorname{SWOT}(\xi)$.

In total, five BFN-QG experiments are run as described previously in each domain: Nadirs, SWOT $(q)$, SWOT $(\xi)$, Nadirs $+\operatorname{SWOT}(q)$ and Nadirs $+\operatorname{SWOT}(\xi)$.

\section{d. Diagnostics for evaluation}

The reconstructed maps are interpolated to the $\left(1 / 60^{\circ} \times\right.$ $1 / 60^{\circ}$ ) grid of the NR at a daily frequency in order to compare them on the basis of the three diagnostics introduced below. Examples of interpolated fields are shown in Fig. 3.

Three evaluation diagnostics are considered, providing integrated and scale-dependent metrics on the algorithm performance. For each of them, a relative score between 0 and 1 is defined to allow an easy comparison.

- The root-mean-square error

The root-mean-square error (RMSE), first computed as a function of time, is defined by

$$
\operatorname{RMSE}(t)=\sqrt{\frac{1}{N} \sum_{i=1}^{N}\left[\operatorname{SSH}(t, i)-\mathrm{SSH}^{\text {true }}(t, i)\right]^{2}},
$$

where $\operatorname{SSH}(t, i)$ and $\operatorname{SSH}^{\text {true }}(t, i)$ are the values of the reconstructed field and the true field at the grid point $i$ and time $t ; N$ is the number of grid points included in the study domain. The RMSE diagnostic is known to be sensitive to phase errors (due to structures misplacement) regardless of the spatial scales of the structures.

For this diagnostic, the relative score $\mathrm{RMSE}_{S}$ is defined by

$$
\operatorname{RMSE}_{S}(t)=1-\frac{\operatorname{RMSE}(t)}{\operatorname{RMS}\left(\operatorname{SSH}^{\text {true }}\right)},
$$

where RMS is the root-mean-square function. For a given time, a score of 1 indicates a perfect reconstruction in terms 
NR
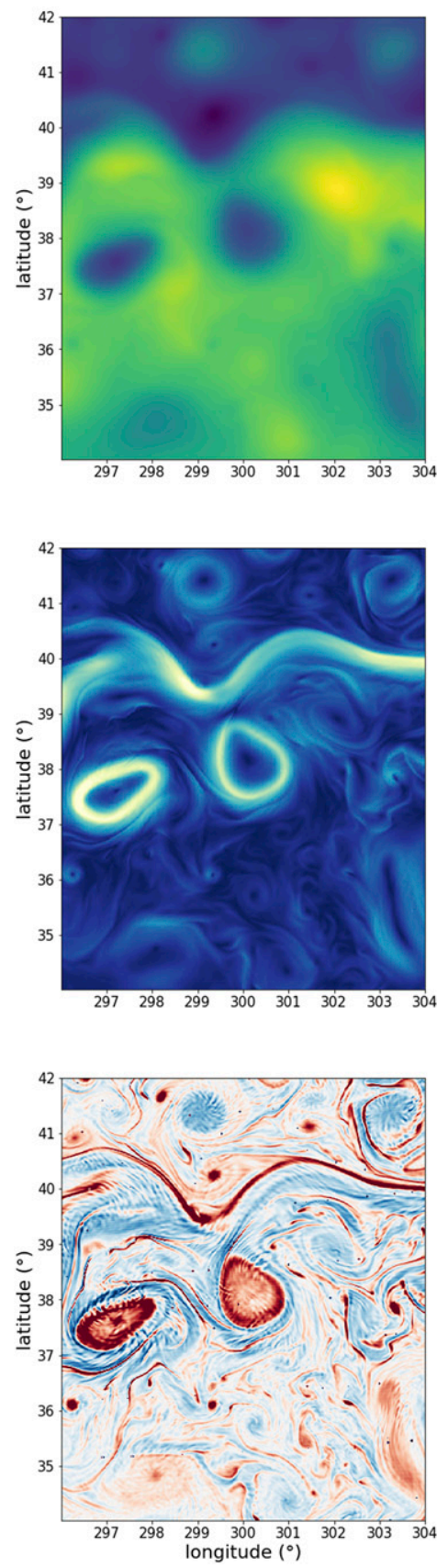

DUACS
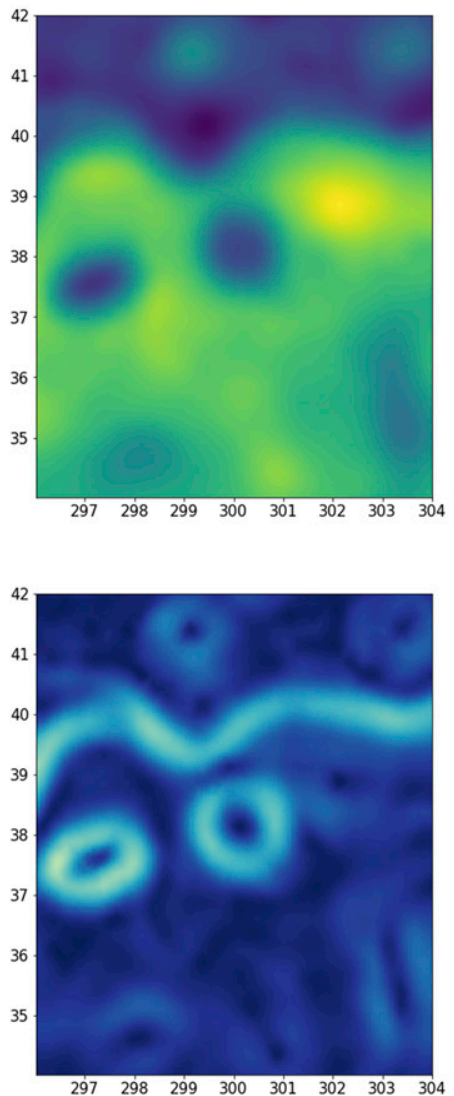

$\begin{array}{llllllll}297 & 298 & 299 & 300 & 301 & 302 & 303 & 304\end{array}$

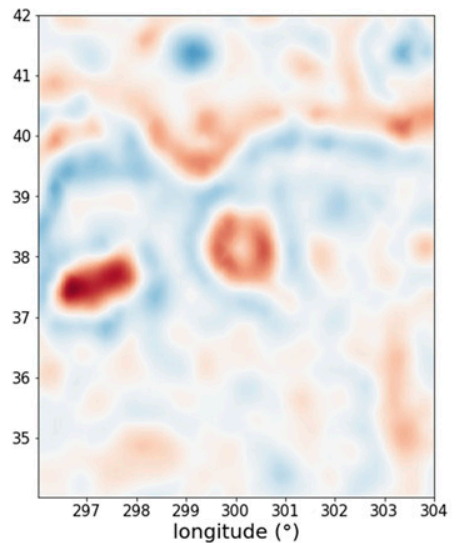

BFN-QG
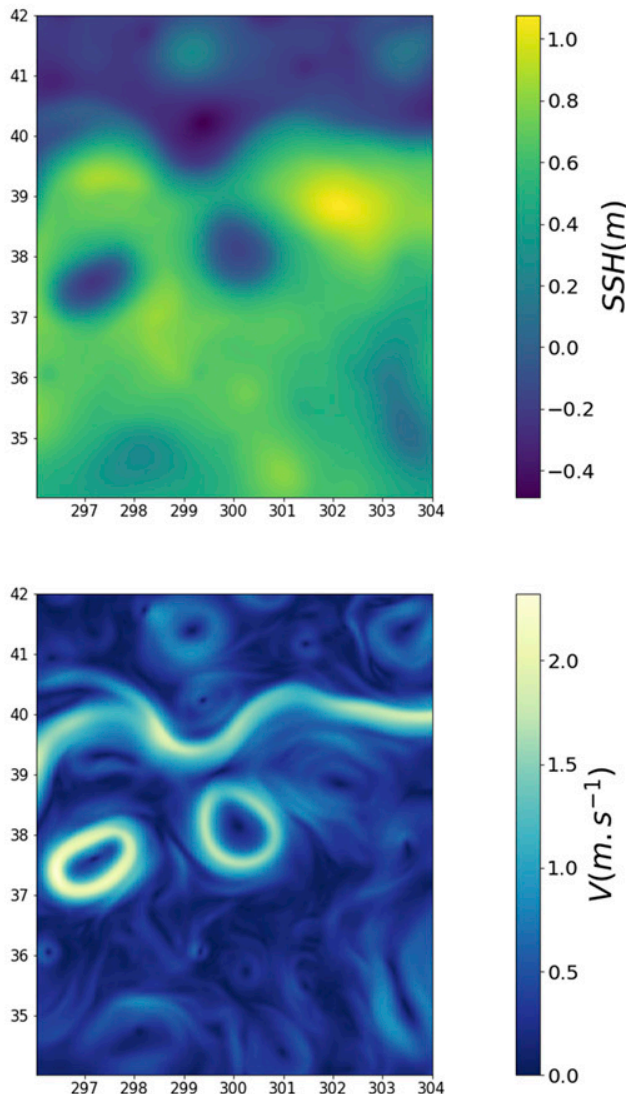

$\begin{array}{llllllll}297 & 298 & 299 & 300 & 301 & 302 & 303 & 304\end{array}$

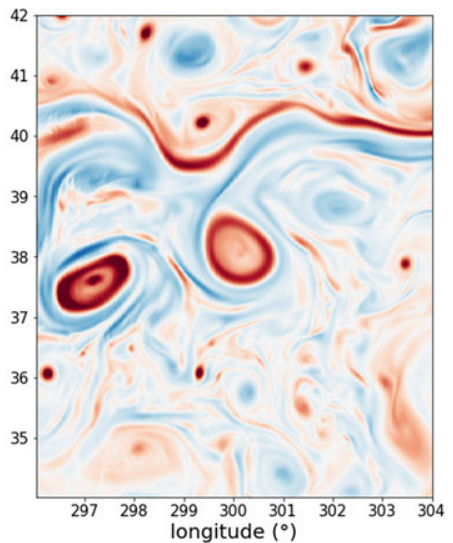

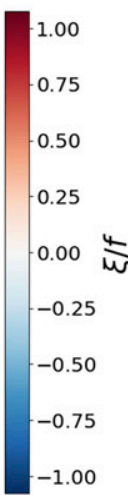

$-1.00$

FIG. 3. Snapshots of (top) SSH, (middle) absolute geostrophic velocity, and (bottom) normalized geostrophic relative vorticity for the (left) NR, (center) DUACS, and (right) BFN-QG on 26 May 2013 in the studied region. DUACS and BFN-QG are processing both nadir and SWOT data. Velocity and relative vorticity maps are computed on the native grids, i.e., before the interpolation to the $\left(1 / 60^{\circ} \times 1 / 60^{\circ}\right)$ grid of the NR.

of RMSE, while a score of 0 indicates that the RMSE is as large as the RMS of the NR.

A time-mean score is also computed, as a single scalar for each experiment.

- The wavenumber power spectral density
This second diagnostic, the wavenumber power spectral density (WPSD) provides a spatial-scale-dependent metric. This is particularly useful to identify the smallest scales resolved by the maps. To compute the WPSD, we use the technique and the tool described in Ajayi et al. (2019). First, 
the SSH fields are detrended and tapered using a Tukey window (with the alpha parameter equal to 0.5 ) along both spatial axes. Then a 2D fast Fourier transform (FFT) is applied to each SSH field. The WPSD is obtained by averaging the 2D FFTs in the azimuthal direction first, and then over time. The score $\mathrm{WPSD}_{S}$ is presented as a function of wavenumber, and defined by

$$
\mathrm{WPSD}_{S}=1-\frac{\mathrm{WPSD}\left(\mathrm{SSH}-\mathrm{SSH}^{\text {true }}\right)}{\operatorname{WPSD}\left(\mathrm{SSH}^{\text {true }}\right)} .
$$

For a given wavenumber, a score of 1 indicates a perfect reconstruction (the amplitude and phase of the corresponding harmonic is right) and a score of 0 indicates no phase correlation. The definition of this score slightly differs from Ballarotta et al. (2019) in that (i) here, the PSD calculation is on the azimuthal direction, and (ii) here, the best score is 1 instead of 0 .

We also define the effective resolution (ER) of a reconstruction by the spatial scale at which WPSD $_{S}$ is equal to 0.5 (Ballarotta et al. 2019). This is also a single scalar, expressed in kilometers, characterizing the spatial resolution of the maps.

- The frequency-wavenumber power spectral density The third diagnostic, the frequency-wavenumber power spectral density (FWPSD), provides a description of the resolved scales both in space and time. Figure 2 depicts the FWPSD of the NR in the studied region over the evaluation time period.

As for the WPSD, the SSH data are first detrended and tapered (here with a Hanning window) in space and time. The FWPSD is then computed by performing the 3D FFT along space and time dimensions. To compact the space dependence to one dimension, the spectra are azimuthally averaged as for WPSD.

Similarly to the WPSD, a score is defined, here in the frequency-wavenumber domain. The formula is similar to that of Eq. (11). This score will be represented on a 2D plot highlighting the reconstruction skills as a function of spatial and temporal scales.

\section{Results and discussion}

The mapping performance of DUACS and BFN-QG techniques are compared in Figs. 3-6 for the five experiments carried out in the studied region. Figure 3 illustrates snapshots of NR and the associated maps reconstructed by DUACS and BFN-QG. Figures 4-6 are presented in a common format: three horizontal panels corresponding to the three diagnostics (see section 3d). For each diagnostic (RMSE, WPSD, and FWPSD), the left panel presents the absolute quantity, and the right panel presents the associated scores. The time-mean RMSEs and the effective resolutions are also reported. NR is plotted in continuous thick black lines, the DUACS products in thin continuous black lines, and the BFN-QG products in thin dash colored lines. Figure 4 refers to the nadir-only scenario, Fig. 5 to the SWOT-only scenario, and Fig. 6 to the joint nadirSWOT scenario. As stated earlier, the two scenarios involving SWOT include two experiments that differ by the way SWOT is assimilated.
In all five experiments, the BFN-QG performs better than DUACS, with one exception for the RMSE when only SWOT is assimilated with the relative vorticity formulation (Fig. 5, top panels).

The BFN-QG outperforms DUACS in the mapping of SSH from nadir altimetry only, and this outperformance is further increased when SWOT is introduced. For instance, the ER of the maps computed with nadir data $(136.7 \mathrm{~km}$ for DUACS and $101.4 \mathrm{~km}$ for the BFN-QG) is improved by $26 \%$ with the BFN-QG in comparison to DUACS (Fig. 4) and this improvement reaches $33 \%$ when adding SWOT data (Fig. 6). This confirms the idea of Ubelmann et al. (2015) that adding information on ocean dynamics should improve the mapping of SSH when wide-swath altimetry is introduced.

In the scenario where only SWOT is assimilated, restricting the nudging to $\xi$ significantly degrades the reconstruction performance. Figure 5 shows a significant increase of the RMSE when nudging only $\xi$. The middle panel of Fig. 5 indicates that this degradation mostly occurs at large spatial scales. In fact, the constraint applied to SSH only comes from the SWOT nadir altimeter through the nudging on the vortex stretching part of the PV and is not sufficient to constrain efficiently the large scales. However, both approaches of BFN-QG improve the ER in comparison with DUACS, what shows the relevance of this method for the reconstruction of scales below $150 \mathrm{~km}$ with SWOT.

In the nadir + SWOT scenarios, the BFN-QG partially damps the time variations of the reconstruction errors in comparison with DUACS, although these variations remain significant. The time variations of the reconstruction errors are clearly visible on the RMSE plots. The increasing phases coincide with time periods without SWOT observations. During these periods, which last approximately 5 days, estimation errors grow due to imperfect dynamics (both QG for BFN-QG and linear interpolation for DUACS). In the SWOT-only scenarios, the error-variation amplitudes in DUACS and BFN-QG results are comparable. Figure 6 shows that the nadir data help to reduce this amplitude with the BFN-QG, more than with DUACS.

The BFN-QG reconstructs structures at small scales with finer details than DUACS, as illustrated in Fig. 3. In particular, the discrepancies between DUACS and BFN-QG appear more significantly on $\xi$ (depicting finer-scale structures) than on SSH. This difference in resolution is confirmed by the frequency-wavenumber spectral diagnostics. According to the FWPSDs, the BFN-QG can reconstruct smaller space and time scales than DUACS in general; in particular, when nadir and SWOT data are accounted for simultaneously, BFN-QG reaches scales of 80-100 km and 10 days. In all scenarios, the energy distribution of the DUACS reconstruction (upper-left panels of frequency-wavenumber PSD sections) is in agreement with the commonly known scales resolved by the maps (Ballarotta et al. 2019), around $120 \mathrm{~km}$ and 12 days. Above these scales (typically above $150 \mathrm{~km}$ and 15 days), the scores (right panels) indicate that all the reconstructions are reliable (blue color) with more signal than errors, except perhaps for the SWOT-only scenarios. Below these scales, three aspects can be highlighted: (i) BFN-QG produces more energy than 

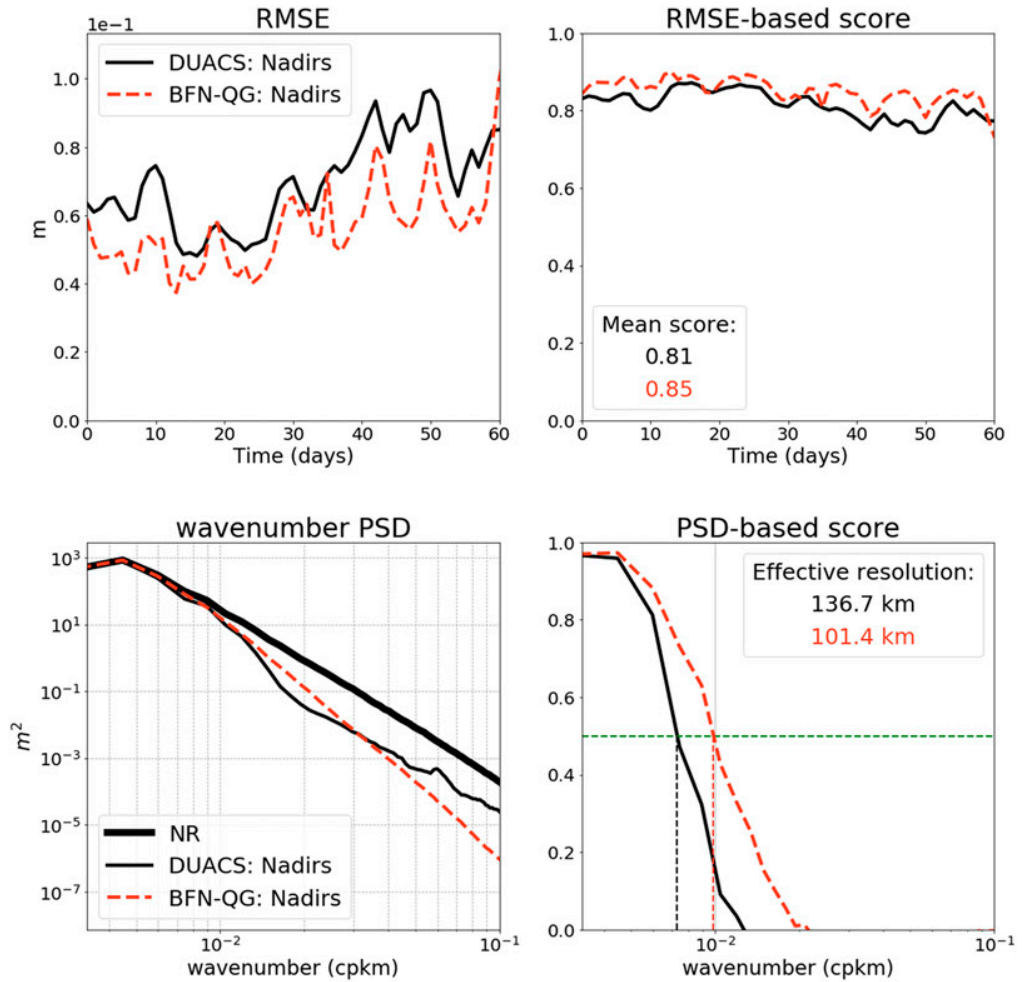

Frequency-wavenumber PSD

DUACS: Nadirs
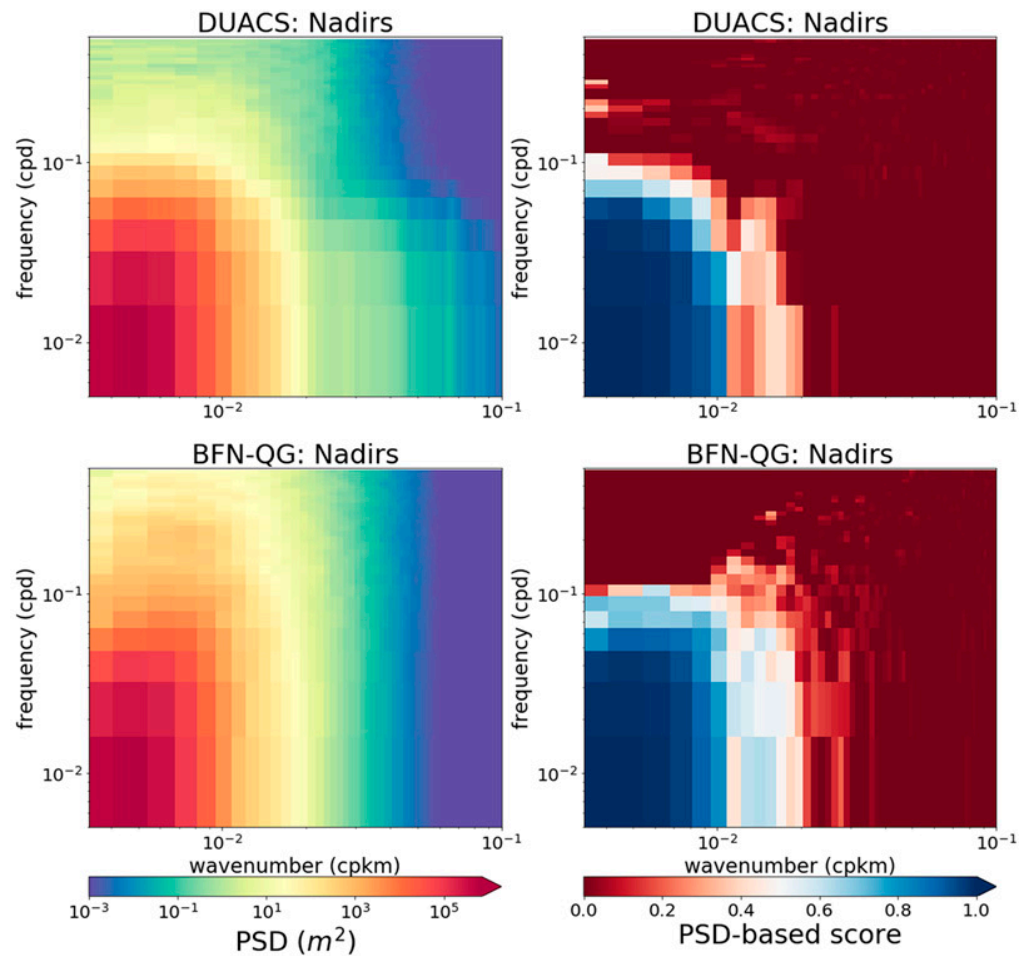

FIG. 4. (top) RMSE, (middle) wavenumber PSD, and (bottom) frequencywavenumber PSD on SSH, for DUACS and BFN-QG processing a constellation of four nadir altimeters in the studied region during the 2-month period. 

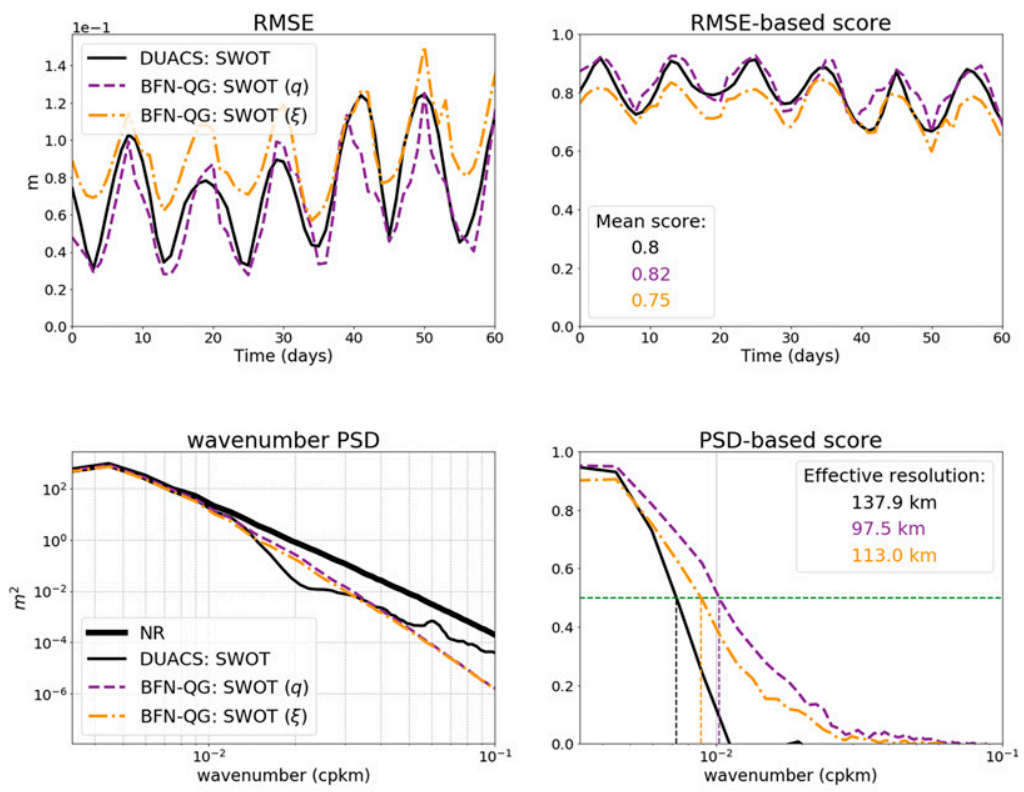

Frequency-wavenumber PSD
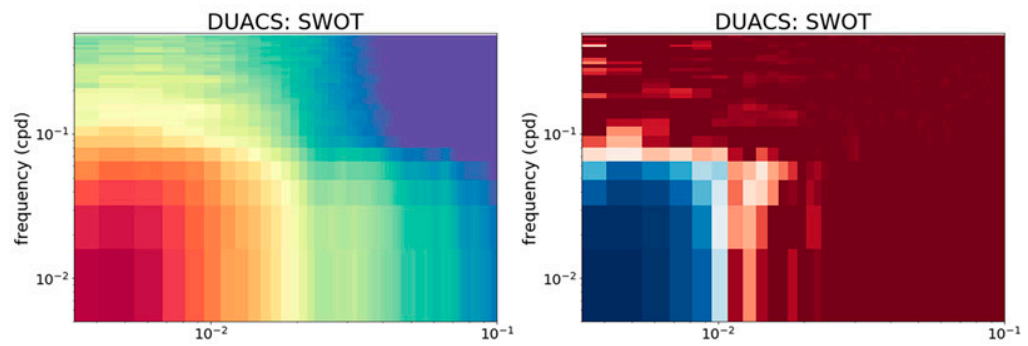

BFN-QG: SWOT $(q)$
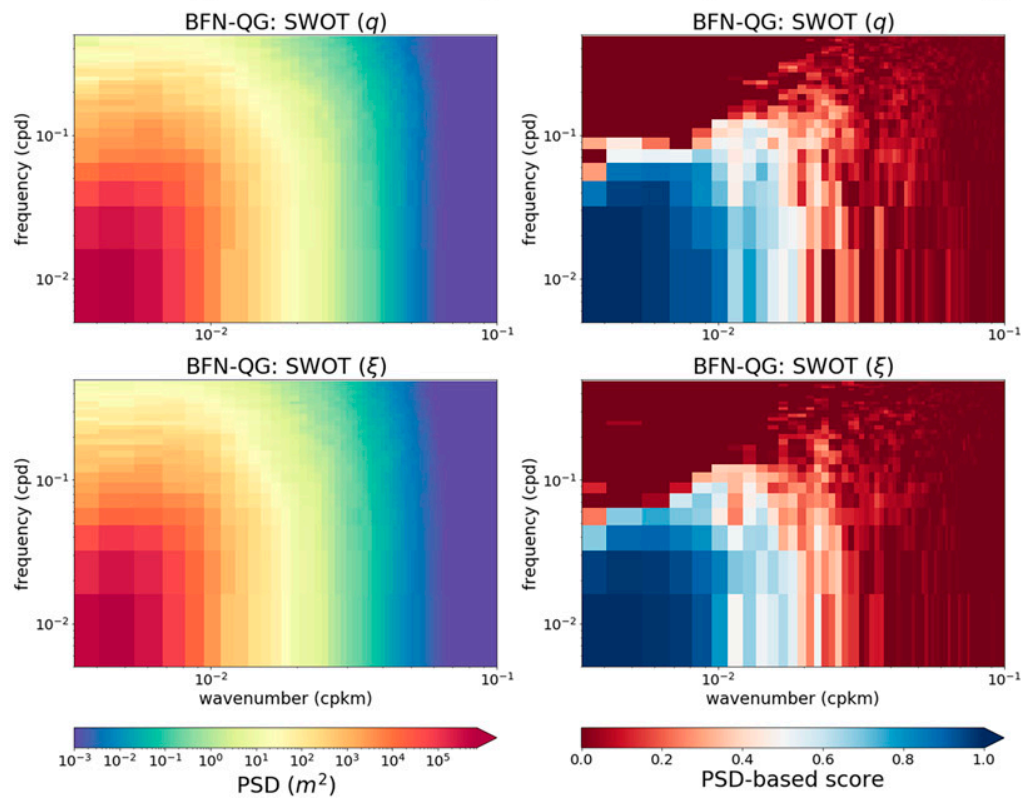

FIG. 5. As in Fig. 4, with SWOT only instead of the nadir altimeters. The BFN-QG is performed by nudging SWOT data on $\mathrm{PV}[\operatorname{SWOT}(q)$, purple dashed line] or on relative vorticity $[\operatorname{SWOT}(\xi)$, orange dashed line]. 

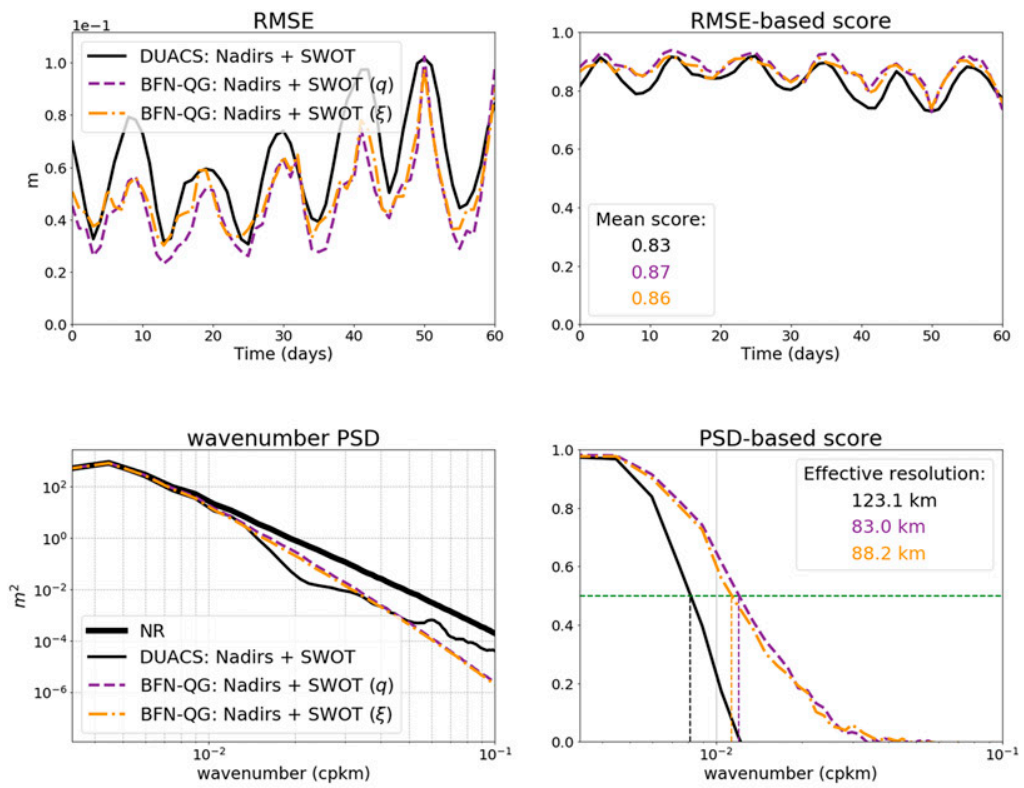

Frequency-wavenumber PSD
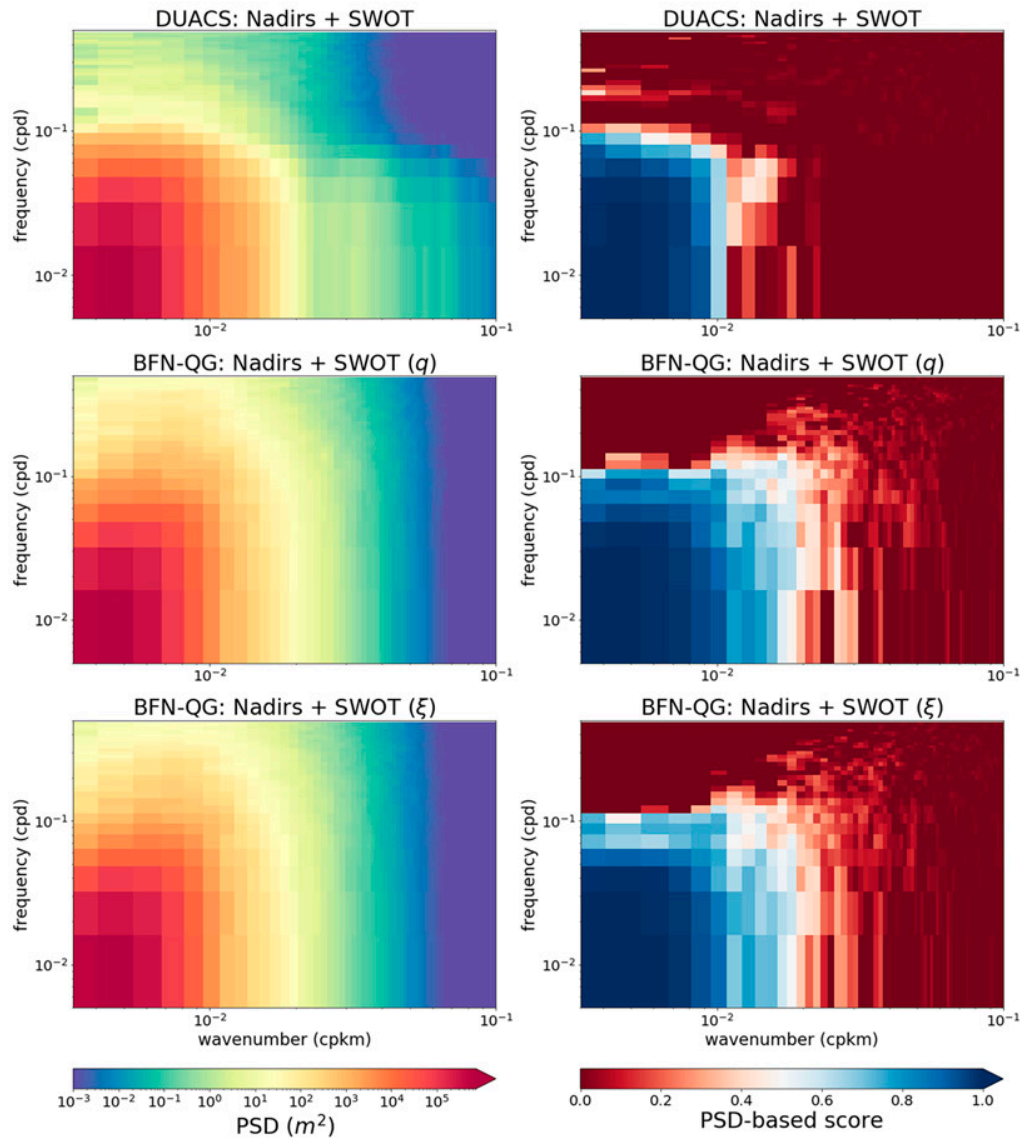

FIG. 6. As in Fig. 5, with SWOT and nadir altimeters altogether. 
DUACS at short times scales (below 10 days), (ii) DUACS and BFN-QG create comparable PSDs at scales above 10 days, and (iii) the scores (right panels) indicate that the reconstructions of scales between 80 and $100 \mathrm{~km}$ are more reliable in BFN-QG than in DUACS. The latter statement remains true for time scales close to 10 days for the Nadir + SWOT scenarios. This is corroborated by Fig. 4 that shows that the discrepancies between DUACS and BFN-QG are much more visible on $\xi$ (depicting finer-scale structures) than on SSH. Note that the spurious energy displayed in the FWPSDs of DUACS at scales above 10 days and below $30 \mathrm{~km}$ is due to the spatial interpolation of DUACS maps on the $\left(1 / 60^{\circ} \times 1 / 60^{\circ}\right) \mathrm{NR}$ grid.

The conclusions on the performances of the mapping methods obtained in this study seem to be robust to geographical region changes. The experiments in another region, namely, the OSMOSIS region, presented in the supplementary material, are very different in terms of mapping challenges to the ones in the region presented here. Despite the difference in the nature of the mapping problem, the improvement brought by the dynamical constraint, i.e., the BFN-QG, in the mapping reconstruction is confirmed in the OSMOSIS region. Although the conclusions might not stand in very specific regions (e.g., tropical or coastal), this result tends to show the robustness of the conclusions to noncoastal midlatitude regions.

\section{Conclusions}

This study aimed at testing the ability of a simple data assimilation technique [viz., the back-and-forth nudging (BFN)] to map sea surface height $(\mathrm{SSH})$ from realistic altimetric observations in the context of the future SWOT mission. The proposed mapping technique uses a 1.5-layer QG model forced by observations of SSH. The model is gradually nudged toward the observations while running forward and backward within a temporal window in an iterative process so as to overcome the problem of time distribution of the observations. The performance of this mapping technique, dubbed the BFN-QG, has been evaluated and compared with the standard SSH mapping products DUACS, for different satellite constellations that include conventional nadir and SWOT altimetric data.

The first conclusion of this work is that the BFN-QG is well suited to interpolate in space and time SSH provided by conventional nadir altimetric data. It manages to both reduce the root-mean-square errors (RMSEs) and improve the effective resolution (ER) of the maps in comparison with the DUACS technique. Future works should test this technique with real (or realistic) altimetric measurements to test the ability of the BFN-QG to process noisy data.

The second conclusion is that the good performance of the BFN-QG is increased when SWOT is added to the constellation of nadirs, improving the space-time resolutions of the maps by a factor comprised between $25 \%$ and $30 \%$ over the DUACS mapping resolutions. It seems that the BFN-QG is even more effective to interpolate the small-scale processes captured by SWOT than the DUACS method. The BFN-QG damps the time variations of reconstruction errors due to irregular satellite sampling better than DUACS. This suggests that using a more complex dynamical model (e.g., a two-layer QG model) could make this process even more efficient.

Finally, if strong spatially correlated errors were to corrupt the SWOT measurement, the BFN-QG allows an alternative formulation that only nudges the relative vorticity derived from the SWOT data which should be less affected by correlated errors. In that context, the results show that this formulation produces relatively good quality SSH reconstructions under the crucial condition that the SWOT data must be combined with nadir data. Future works should expend this study by testing the BFN-QG with noisy synthetic SWOT data.

Of course, many developments remain to be made before the efficient mapping of SSH from SWOT. Some have been suggested previously. The mapping methods must be tested with more realistic error-containing observations. More sophisticated dynamical models may further improve the mapping.

A specific limitation of the work exposed in this paper lies in the assumption of a fixed deformation radius in the model, associated with the small size of the ocean domains considered. For SSH reconstructions over wider domains, we would have to deal with space-and-time variations (Guinehut et al. 2012) of the deformation radius and its compatibility with the QG model assumptions.

One particularly challenging development is related to the presence of internal gravity waves (IGWs) in the ocean. To maintain the focus of this study to a proof of concept, the Nature Run (NR) did not simulate IGWs therefore the impact of IGWs on SSH reconstruction was not a concern. However, it is strongly suspected that IGWs will affect the signal measured by SWOT. Even though, in many regions, IGWs should not be the dominating component of the signal at the scales reconstructed by the BFN-QG system, their impact on $\mathrm{SSH}$ reconstruction should be considered in further studies. For instance, an interesting idea would be to extend the BFN approach to IGWs by replacing the QG model with a gravity wavepermitting model.

Another challenging development is how to extend dynamical mapping approaches such as BFN-QG to coastal and tropical regions. Once again, a possible development would be to replace the QG model with a model more appropriate to simulate the dynamics of these regions. One particular limitation of this task is that the employed reduced models have to be reversible to work with $\mathrm{BFN}$.

Acknowledgments. The authors thank Jean Marc Molines for carrying out the NATL60 simulation and Aurélie Albert from Ocean Next for her contribution to the preparation and extraction of the data. This research was funded by ANR (Project ANR-17-CE01-0009-01) and CNES through the SWOT Science Team program.

Data availability statement. The data used in this study can be accessed at the following URL: https://ige-meom-opendap. univ-grenoble-alpes.fr/thredds/catalog/meomopendap/extract/ LeGuillou/data_LeGuillou_2020/catalog.html. An SSH mapping data challenge, based on the experimental setup presented in this paper, is described here: https://github.com/ocean-datachallenges/2020a_SSH_mapping_NATL60 and identified on 
Zenodo platform with DOI 10.5281/zenodo.4045400 (https:// doi.org/10.5281/zenodo.4045400).

\section{REFERENCES}

Ablain, M., J. F. Legeais, P. Prandi, M. Marcos, L. Fenoglio-Marc, H. B. Dieng, J. Benveniste, and A. Cazenave, 2017: Satellite altimetry-based sea level at global and regional scales. Surv. Geophys., 38, 7-31, https://doi.org/10.1007/s10712-016-9389-8.

Ajayi, A., J. Le Sommer, E. Chassignet, J.-M. Molines, X. Xu, A. Albert, and W. Dewar, 2019: Diagnosing cross-scale kinetic energy exchanges from two submesoscale permitting ocean models. Earth Space Science Open Archive, https://doi.org/ 10.1002/essoar.10501077.1.

,,,,,------ , and E. Cosme, 2020: Spatial and temporal variability of the North Atlantic eddy field from two kilometric-resolution ocean models. J. Geophys. Res. Oceans, 125, e2019JC015827, https://doi.org/10.1029/2019JC015827.

Amores, A., G. Jordà, T. Arsouze, and J. L. Sommer, 2018: Up to what extent can we characterize ocean eddies using presentday gridded altimetric products? J. Geophys. Res. Oceans, 123, 7220-7236, https://doi.org/10.1029/2018JC014140.

Anthes, R., 1974: Data assimilation and initialization of hurricane prediction models. J. Atmos. Sci., 31, 702-719, https://doi.org/ 10.1175/1520-0469(1974)031<0702:DAAIOH >2.0.CO;2.

Auroux, D., and J. Blum, 2008: A nudging-based data assimilation method: The back and forth nudging (BFN) algorithm. Nonlinear Processes Geophys., 15, 305-319, https://doi.org/ 10.5194/npg-15-305-2008.

Ballarotta, M., and Coauthors, 2019: On the resolutions of ocean altimetry maps. Ocean Sci., 15, 1091-1109, https://doi.org/ 10.5194/os-15-1091-2019.

—_, and Coauthors, 2020: Dynamic mapping of along-track ocean altimetry: Performance from real observations. J. Atmos. Oceanic Technol., 37, 1593-1601, https://doi.org/10.1175/ JTECH-D-20-0030.1.

Blayo, E., J. Verron, and J. M. Molines, 1994: Assimilation of TOPEX/Poseidon altimeter data into a circulation model of the North Atlantic. J. Geophys. Res., 99, 24 691-24 705, https:// doi.org/10.1029/94JC01644.

Buongiorno Nardelli, B., 2020: A multi-year timeseries of observationbased 3D horizontal and vertical quasi-geostrophic global ocean currents. Earth Sys. Sci. Data, 12, 1711-1723, https://doi.org/ 10.5194/essd-12-1711-2020.

Chelton, D. B., R. A. deSzoeke, M. G. Schlax, K. El Naggar, and N. Siwertz, 1998: Geographical variability of the first baroclinic Rossby radius of deformation. J. Phys. Oceanogr., 28, 433-460, https://doi.org/10.1175/1520-0485(1998)028<0433: GVOTFB $>2.0 . \mathrm{CO} ; 2$.

—, M. G. Schlax, and R. M. Samelson, 2011: Global observations of nonlinear mesoscale eddies. Prog. Oceanogr., 91, 167-216, https://doi.org/10.1016/j.pocean.2011.01.002.

Fresnay, S., A. L. Ponte, S. Le Gentil, and J. Le Sommer, 2018: Reconstruction of the 3-D dynamics from surface variables in a high-resolution simulation of North Atlantic. J. Geophys. Res. Oceans, 123, 1612-1630, https://doi.org/10.1002/2017JC013400.

Fu, L.-L., D. B. Chelton, P.-Y. Le Traon, and R. Morrow, 2010: Eddy dynamics from satellite altimetry. Oceanography, 23 (4), 14-25, https://doi.org/10.5670/oceanog.2010.02.

—-, E. Rodriguez, D. Alsdorf, and R. Morrow, 2012: The SWOT mission science document. NASA JPL Tech. Rep., 222 pp., https://swot.jpl.nasa.gov/files/swot/SWOT_MSD_ 1202012.pdf.
Gaspari, G., and S. E. Cohn, 1999: Construction of correlation functions in two and three dimensions. Quart. J. Roy. Meteor. Soc., 125, 723-757, https://doi.org/10.1002/qj.49712555417.

Gaultier, L., C. Ubelmann, and L.-L. Fu, 2016: The challenge of using future SWOT data for oceanic field reconstruction. J. Atmos. Oceanic Technol., 33, 119-126, https://doi.org/ 10.1175/JTECH-D-15-0160.1.

Gómez-Navarro, L., R. Fablet, E. Mason, A. Pascual, B. Mourre, E. Cosme, and J. Le Sommer, 2018: SWOT spatial scales in the western Mediterranean Sea derived from pseudo-observations and an ad hoc filtering. Remote Sens., 10, 599, https://doi.org/ 10.3390/rs10040599.

Guinehut, S., A.-L. Dhomps, G. Larnicol, and P.-Y. Le Traon, 2012: High resolution 3-D temperature and salinity fields derived from in situ and satellite observations. Ocean Sci., 8, 845857, https://doi.org/10.5194/os-8-845-2012.

Hoke, J. E., and R. A. Anthes, 1976: The initialization of numerical models by a dynamic-initialization technique. Mon. Wea. Rev., 104, 1551-1556, https://doi.org/10.1175/1520-0493(1976)104<1551: TIONMB $>2.0 . \mathrm{CO} ; 2$.

Holland, W. R., and P. Malanotte-Rizzoli, 1989: Assimilation of altimeter data into an ocean circulation model: Space versus time resolution studies. J. Phys. Oceanogr., 19, 1507-1534, https://doi.org/10.1175/1520-0485(1989)019<1507: AOADIA $>2.0 . \mathrm{CO} ; 2$.

Lea, D. J., J.-P. Drecourt, K. Haines, and M. J. Martin, 2008: Ocean altimeter assimilation with observational- and model-bias correction. Quart. J. Roy. Meteor. Soc., 134, 1761-1774, https://doi.org/10.1002/qj.320.

Leeuwenburgh, O., 2005: Assimilation of along-track altimeter data in the tropical Pacific region of a global OGCM ensemble. Quart. J. Roy. Meteor. Soc., 131, 2455-2472, https:// doi.org/10.1256/qj.04.146.

Le Traon, P. Y., F. Nadal, and N. Ducet, 1998: An improved mapping method of multisatellite altimeter data. J. Atmos. Ocean. Technol., 15, 522-534, https://doi.org/10.1175/15200426(1998)015<0522:AIMMOM>2.0.CO;2.

Martin, M. J., A. Hines, and M. J. Bell, 2007: Data assimilation in the foam operational short-range ocean forecasting system: A description of the scheme and its impact. Quart. J. Roy. Meteor. Soc., 133, 981-995, https://doi.org/10.1002/qj.74.

Metref, S., E. Cosme, J. Le Sommer, N. Poel, J.-M. Brankart, J. Verron, and L. Gómez Navarro, 2019: Reduction of spatially structured errors in wide-swath altimetric satellite data using data assimilation. Remote Sens., 11, 1336, https://doi.org/ 10.3390/rs11111336.

,,-- F. Le Guillou, J. Le Sommer, J.-M. Brankart, and J. Verron, 2020: Wide-swath altimetric satellite data assimilation with correlated-error reduction. Front. Mar. Sci., 6, 822, https://doi.org/10.3389/fmars.2019.00822.

Molines, J.-M., 2018: meom-configurations/NATL60-CJM165: NATL60 code used for CJM165 experiment. Zenodo, https:// zenodo.org/record/1210116\#.Xp2bwFMzYUE.

Morrow, R., and P. Le Traon, 2012: Recent advances in observing mesoscale ocean dynamics with satellite altimetry. Adv. Space Res., 50, 1062-1076, https://doi.org/10.1016/ j.asr.2011.09.033.

_ - and Coauthors, 2019: Global observations of fine-scale ocean surface topography with the Surface Water and Ocean Topography (SWOT) mission. Front. Mar. Sci., 6, 232, https:// doi.org/10.3389/fmars.2019.00232.

Mulet, S., M.-H. Rio, A. Mignot, S. Guinehut, and R. Morrow, 2012: A new estimate of the global 3D geostrophic ocean circulation 
based on satellite data and in-situ measurements. Deep-Sea Res. II, 77-80, 70-81, https://doi.org/10.1016/j.dsr2.2012.04.012.

Pascual, A., Y. Faugère, G. Larnicol, and P.-Y. L. Traon, 2006: Improved description of the ocean mesoscale variability by combining four satellite altimeters. Geophys. Res. Lett., 33, L02611, https://doi.org//10.1029/2005GL024633.

Peral, E., and D. Esteban-Fernandez, 2018: SWOT mission performance and error budget. IEEE Int. Geoscience and Remote Sensing Symp., Valencia, Spain, IEEE, 8625-8628, https:// doi.org/10.1109/IGARSS.2018.8517385.

Ruggiero, G. A., Y. Ourmieres, E. Cosme, J. Blum, D. Auroux, and J. Verron, 2015: Data assimilation experiments using diffusive back-and-forth nudging for the NEMO ocean model. Nonlinear Processes Geophys., 22, 233-248, https://doi.org/10.5194/npg-22233-2015.

Skamarock, W., 2004: Evaluating mesoscale NWP models using kinetic energy spectra. Mon. Wea. Rev., 132, 3019-3032, https://doi.org/10.1175/MWR2830.1.

Storto, A., S. Dobricic, S. Masina, and P. Di Pietro, 2011: Assimilating along-track altimetric observations through local hydrostatic adjustment in a global ocean variational assimilation system. Mon. Wea. Rev., 139, 738-754, https://doi.org/ 10.1175/2010MWR3350.1.

Taburet, G., and Coauthors, 2019: DUACS DT2018: 25 years of reprocessed sea level altimetry products. Ocean Sci., 15, 12071224, https://doi.org/10.5194/os-15-1207-2019.

Ubelmann, C., P. Klein, and L.-L. Fu, 2015: Dynamic interpolation of sea surface height and potential applications for future high-resolution altimetry mapping. J. Atmos. Ocean. Technol., 32, 177-184, https://doi.org/10.1175/JTECH-D-14-00152.1.

— B. Cornuelle, and L.-L. Fu, 2016: Dynamic mapping of alongtrack ocean altimetry: Method and performance from observing system simulation experiments. J. Atmos. Ocean. Technol., 33, 1691-1699, https://doi.org/10.1175/JTECH-D15-0163.1.

Vallis, G. K., 2006: Atmospheric and Oceanic Fluid Dynamics: Fundamentals and Large-Scale Circulation. Cambridge University Press, 773 pp.

Verron, J., 1990: Altimeter data assimilation into an ocean circulation model-Sensitivity to orbital parameters. J. Geophys. Res., 95, 11 443-11 459, https://doi.org/10.1029/JC095iC07p11443.

_ 1992: Nudging satellite altimeter data into quasi-geostrophic ocean models. J. Geophys. Res., 97, 7479-7491, https://doi.org/ 10.1029/92JC00200.

von Schuckmann, K., et al., 2018: Copernicus Marine Service ocean state report. J. Oper. Oceanogr., 11, S1-S142, https://doi.org/ 10.1080/1755876X.2018.1489208.

Wan, L., L. Bertino, and J. Zhu, 2010: Assimilating altimetry data into a HYCOM model of the Pacific: Ensemble optimal interpolation versus ensemble Kalman filter. J. Atmos. Ocean. Technol., 27, 753-765, https://doi.org/10.1175/2009JTECHO626.1.

Wang, J., L.-L. Fu, H. S. Torres, S. Chen, B. Qiu, and D. Menemenlis, 2019: On the spatial scales to be resolved by the surface water and ocean topography Ka-band radar interferometer. J. Atmos. Oceanic Technol., 36, 87-99, https://doi.org/10.1175/JTECH-D18-0119.1. 